\title{
Application and Efficacy of Super-Magnifying Endoscopy for the Lower Intestinal Tract
}

\author{
Naoki Hosoe and Haruhiko Ogata \\ Center for Diagnostic and Therapeutic Endoscopy, Keio University School of Medicine, Tokyo, Japan
}

Endoscopy plays a significant role in the diagnosis, management, and surveillance of colorectal cancer (CRC) and inflammatory bowel diseases (IBDs). Moreover, magnifying endoscopy and image-enhanced endoscopy has a crucial role in the clinical setting. Recently, a super-magnifying endoscope has been developed, and two devices, confocal laser endomicroscopy (CLE) and an endocytoscopy system (ECS), which allow in vivo microscopic inspection of the microstructural mucosal features of the gastrointestinal tract, are currently available. Studies on the use of ECS in CRC were reported by a Japanese group. Additionally, a few studies on the use of ECS in IBD have been reported. CLE has been shown to be reliable in assessing the activity of the disease in IBDs in both ulcerative colitis and Crohn's disease. Various published studies evaluated the use of CLE during colonoscopy to distinguish colorectal polyp pathology and neoplasia. However, these studies are heterogeneous, and further evidence is necessary to confirm the efficacy of CLE. Clin Endosc 2016;49:37-40

Key Words: Super-magnifying endoscopy; Endocytoscopy; Confocal laser endomicroscopy; Magnifying endoscopy

\section{INTRODUCTION}

Endoscopy plays a significant role in the diagnosis, management, and surveillance of colorectal cancer (CRC) and inflammatory bowel diseases (IBDs). Moreover, magnifying endoscopy ${ }^{1,2}$ and image-enhanced endoscopy ${ }^{3}$ have a crucial role in the clinical setting. Recently, a super-magnifying endoscope has been developed, and two devices, confocal laser endomicroscopy (CLE; Mauna Kea Technologies, Paris, France; and Pentax, Tokyo, Japan) and an endocytoscopy system (ECS; Olympus, Tokyo, Japan), which allow in vivo microscopic observation of the microstructural mucosa of the gastrointestinal tract, are currently available. These devices are used in

Received: September 23, 2015 Revised: November 1, 2015

Accepted: November 2, 2015

Correspondence: Naoki Hosoe

Center for Diagnostic and Therapeutic Endoscopy, Keio University School of Medicine, 35 Shinanomachi, Shinjuku, Tokyo 160-8582, Japan

Tel: +81-3-3353-1211, Fax: +81-3-3357-2778, E-mail: nhosoe@keio.jp

(c) This is an Open Access article distributed under the terms of the Creative Commons Attribution Non-Commercial License (http://creativecommons.org/ licenses/by-nc/3.0) which permits unrestricted non-commercial use, distribution, and reproduction in any medium, provided the original work is properly cited. clinical studies rather than in clinical practice. In this review article, we focused on the application and efficacy of these newly developed devices in the lower intestinal tract.

\section{ENDOCYTOSCOPY}

ECS is based on the principle of contact light microscopy. ECS observation also requires pretreatment with methylene blue or toluidine blue staining. ${ }^{4}$ ECS has two types of endoscope: a probe-based ECS and an integrated scope-type ECS (iECS). The iECS has adjustable scope hardness with an outside diameter of $13.6 \mathrm{~mm}$ at the distal end and a working length of $1330 \mathrm{~mm} .{ }^{5}$ The first-generation iECS has a dual-charged coupled device (CCD, prototype, XCF-260EC1; Olympus). This scope can perform conventional and super-magnifying endoscopic observation consecutively using one-touch switch operation without changing the endoscope. In the ECS mode, this scope has a magnification capability of $\times 450$, with a depth of field if $50 \mu \mathrm{m}$ and a field of view of $400 \times 400 \mu \mathrm{m}$. The second-generation iECS is a single-CCD integrated type (prototype, CF-Y0020-I; Olympus). It has a $\times 380$ magnification, with a focusing depth of $50 \mu \mathrm{m}$ and a field of view of $700 \times 600$ 
$\mu \mathrm{m}$, which can obtain super-magnification with narrow band imaging (NBI) pictures. ${ }^{6}$

\section{APPLICATION OF ENDOCYTOSCOPY SYSTEM FOR COLORECTAL CANCER}

Several studies on the application of ECS in CRC were reported by a Japanese group. Kudo et al. ${ }^{5}$ created an endocytoscopic (EC) classification for colonic neoplasia using iECS. They classified the normal mucosa as ECla, all hyperplastic polyps as EC1b, dysplasias as EC2, and massively invasive submucosal cancers (SMm) or worse as EC3b. In that pilot study, they showed a sensitivity of $100 \%$ and a specificity of $100 \%(p<0.05)$ in differentiating nonneoplastic from neoplastic lesions. They also showed a high sensitivity (90.1\%) and a high specificity (99.2\%) in differentiating "SMm or worse" from other neoplastic lesions. Mori et al. ${ }^{7}$ validated the EC classification using a randomized, controlled, open-label trial to determine the noninferiority of the first-generation iECS to standard biopsy. They showed that the diagnostic accuracy of iECS for the discrimination of neoplastic lesions was $94.1 \%$ (95\% confidence interval [CI], 87.6 to 97.8), whereas that of standard biopsy was $96.0 \%$ (95\% CI, 90.2 to 98.9$)$. These results were within the noninferiority margin (absolute difference, $-1.9 \% ; 8.6 \%$ to $5.0 \%$ ). They concluded that iECS was noninferior to standard biopsy in discriminating neoplastic lesions. In a recent study, Kudo et al. ${ }^{8}$ focused on the microvasculature of colonic neoplasia. They evaluated 198 consecutive colonic lesions using second-generation iECS with NBI. They classified the EC vascular (EC-V) pattern into three categories: EC-V1, obscure surface microvessels; EC-V2, clearly observed surface microvessels of a uniform caliber and arrangement; EC-V3, dilated surface microvessels of nonhomogeneous caliber or arrangement. They showed that the sensitivity, specificity, and accuracy of the EC-V1 pattern for diagnosing hyperplastic polyps were $95.5 \%, 99.4 \%$, and $99.0 \%$, respectively, whereas those of the EC-V3 pattern for diagnosing invasive cancer were $74.6 \%, 97.2 \%$, and $88.6 \%$, respectively. They concluded that iECS could evaluate the microvasculature of colorectal lesions, and the EC-V pattern diagnosis was highly correlated with the histopathological diagnoses of other optical biopsy modalities.

\section{APPLICATION OF ENDOCYTOSCOPY SYSTEM FOR INFLAMMATORY BOWEL DISEASE}

A few studies concerning the use of ECS in IBDs have been reported. The efficacy of ECS in evaluating colonic inflammation has not yet been confirmed. We used ECS to diagnose amoebic colitis, an infectious type of colitis, in five patients. ${ }^{9}$ We directly detected Entamoeba histolytica trophozoites in all five cases (100\%). In contrast, serology only detected three patients (60\%) positive for the disease, and hematoxylin and eosin staining showed three positive histological findings as well. We also applied ECS to ulcerative colitis (UC). We created an ECS score (ECSS) to determine the histopathological activity index of UC. ${ }^{10}$ We used first-generation iECS with $\times 450$ magnification, and sample biopsies were obtained from the same site. To validate the ECSS, we calculated the correlation between the ECSS and Matts' histopathological grade, which was determined by an expert pathologist. The ECSS of UC consists of the sum of the indices for shape (0 to 3), the distance between crypts ( 0 to 2 ), and the visibility of superficial microvessels (0 to 1). ECSS scores showed a strong correlation with Matts' histopathological grades $(\rho=0.713, p<0.001)$. Recently, another group ${ }^{11}$ evaluated the clinical usefulness of ECSS in accurately monitoring UC during remission. They classified enrolled patients into two groups: those with an ECSS of 0 to 2 (grade A) and those with an ECSS of 3 to 6 (grade B). They reported that the relapse rate of grade B was significantly greater than that of grade A. They concluded that the ECSS might be a predictive indicator of UC relapse.

\section{CONFOCAL LASER ENDOMICROSCOPY}

CLE was first reported in 2004. Recently, it can be performed with two devices: one integrated into the endoscope (iCLE; Pentax) and another using a mini-probe through the scope (pCLE, Cellvizio; Mauna Kea Technologies) (Fig. 1). ${ }^{12}$ The pCLE consists of a laser scanning unit (Fig. 1A) and a mini-probe (Fig. 1B). The mini-probe can be advanced through the working channel of a standard endoscope. CLE is based on tissue illumination with a low-power laser, with subsequent detection of the fluorescence of light reflected from the tissue. Confocal imaging can be based on tissue reflectance or fluorescence. Fluorescent contrast agents are most commonly used. Fluorescein sodium (fluorescein sodium, AK Fluor; Akorn Pharmaceutical, Lake Forest, IL, USA) is intravenously administered for CLE observation, ${ }^{13}$ or acriflavine (Sigma Pharmaceuticals, Rowville, Australia), tetracycline, or cresyl violet (AnaSpec Inc., San Jose, CA, USA) are topically sprayed. $^{14}$

\section{APPLICATION OF CONFOCAL LASER ENDOMICROSCOPY FOR COLORECTAL CANCER AND POLYP}

Various published studies evaluated the use of CLE during colonoscopy to distinguish colorectal polyp pathology and 

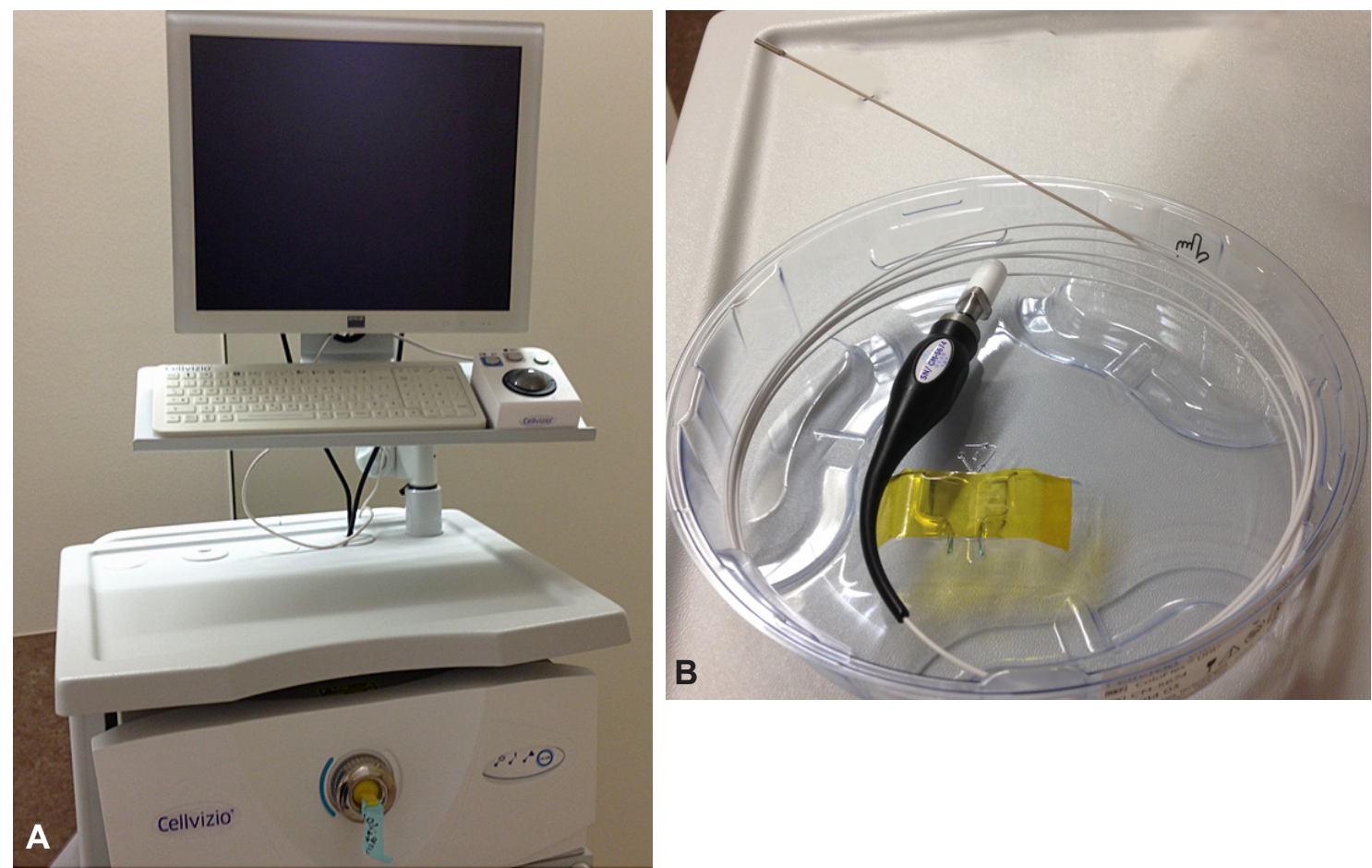

Fig. 1. Probe-based confocal laser endomicroscopy system. (A) Laser scanning unit. (B) Mini-probe. The mini-probe can be advanced through the working channel of a standard endoscope.

Table 1. Performance of Confocal Laser Endomicroscopy for Colonic Neoplasia

\begin{tabular}{|c|c|c|c|c|c|c|}
\hline Study & No. of subjects & Sensitivity, \% & Specificity, \% & PPV, \% & NPV, \% & Accuracy, \% \\
\hline Kiesslich et al. $(2004)^{15}$ & 42 & 97.4 & 99.4 & - & - & 99.2 \\
\hline Meining et al. $(2007)^{16}$ & 13 & 93 & 92.0 & - & - & - \\
\hline Hurlstone et al. $(2008)^{17}$ & 40 & 97.4 & 97.4 & - & - & 99.1 \\
\hline Sanduleanu et al. $(2010)^{18}$ & 72 & 97.3 & 92.8 & - & - & 95.7 \\
\hline De Palma et al. $(2010)^{19}$ & 20 & 100 & 84.6 & 90.5 & 100 & 92.3 \\
\hline Buchner et al. $(2010)^{20}$ & 75 & 91 & 76 & - & - & - \\
\hline Shahid et al. $(2012)^{21}$ & 65 & 86 & 78 & 76 & 88 & 82 \\
\hline Shahid et al. $(2012)^{22}$ & 92 & 97 & 77 & 55 & 99 & 81 \\
\hline Gómez et al. $(2013)^{23}$ & 147 & 43.4 & 70.6 & 18.6 & 89.0 & - \\
\hline
\end{tabular}

PPV, positive predictive value; NPV, negative predictive value.

neoplasia (Table 1). ${ }^{15-23}$ However, these studies are heterogeneous. The sensitivity of CLE in distinguishing colorectal polyp pathology ranges from $43.4 \%$ to $100 \%$. These studies are difficult to compare because of differing primary objectives and modality of CLE used (iCLE vs. pCLE). We need more evidence to be confirm the efficacy of CLE.

\section{APPLICATION OF CONFOCAL LASER ENDOMICROSCOPY FOR INFLAMMATORY BOWEL DISEASE}

CLE has been shown to assess the activity of the disease reliably in IBD, ${ }^{24}$ both in UC and Crohn's disease. Neumann et al. $^{25}$ developed a score named the Crohn's Disease Endomicroscopic Activity Score (CDEAS) to assess in vivo real-time disease activity. CDEAS consists of six parameters: crypt number (increased or decreased), crypt distortion, microerosions, cellular infiltrate, vascularity, and number of goblet cells (increased or decreased). By assigning 1 point for each given parameter, the score ranges from 0 to 8 . They showed that CDEAS strongly correlated with the C-reactive protein level. Li et $\mathrm{al}^{26}$ assessed the potential of CLE in the grading of UC activity. They focused on three categories: crypt architecture, microvascular alterations, and fluorescein leakage. They clas- 
sified crypt architecture into four grades, A to D: A, regular arrangement; B, irregular; C, dilation of crypt openings; D, crypt destruction and/or crypt abscess. They confirmed that crypt architecture classification was strongly correlated with histological index.

\section{FUTURE PERSPECTIVE}

Super-magnifying endoscopy, including ECS and CLE, is mainly used in upper and lower intestinal diseases. Residual feces and/or mucous sometimes disturb optimal super-magnifying observation, and with the presence of a small amount of feces and mucus in the small intestine, the small intestinal circumference is appropriate for super-magnifying observation. We have started a pilot study using single-balloon enteroscopy and CLE to observe small intestinal diseases. In the near future, the use of super-magnifying endoscopy would be reported.

\section{CONCLUSIONS}

Super-magnifying endoscopy, including ECS and CLE, has the potential ability to obtain real-time in vivo histopathology.

\section{Conflicts of Interest}

The authors have no financial conflicts of interest.

\section{REFERENCES}

1. Iwatate M, Sano Y, Hattori S, et al. The addition of high magnifying endoscopy improves rates of high confidence optical diagnosis of colorectal polyps. Endosc Int Open 2015;3:E140-E145.

2. Oka S, Tanaka S, Chayama K. Detection of nonpolypoid colorectal neoplasia using magnifying endoscopy in colonic inflammatory bowel disease. Gastrointest Endosc Clin N Am 2014;24:405-417.

3. Santos CE, Pereira-Lima JC, Lopes CV, Malaman D, Parada AA, Salomão $\mathrm{AD}$. Comparative study between MBI (FICE) and magnification chromoendoscopy with indigo carmine in the differential diagnosis of neoplastic and non-neoplastic lesions of the colorectum. Arq Gastroenterol 2009;46:111-115.

4. Neumann H, Fuchs FS, Vieth M, et al. Review article: in vivo imaging by endocytoscopy. Aliment Pharmacol Ther 2011;33:1183-1193.

5. Kudo SE, Wakamura K, Ikehara N, Mori Y, Inoue H, Hamatani S. Diagnosis of colorectal lesions with a novel endocytoscopic classification: a pilot study. Endoscopy 2011;43:869-875.

6. Kutsukawa M, Kudo SE, Ikehara N, et al. Efficiency of endocytoscopy in differentiating types of serrated polyps. Gastrointest Endosc 2014;79:648-656.

7. Mori Y, Kudo S, Ikehara N, et al. Comprehensive diagnostic ability of endocytoscopy compared with biopsy for colorectal neoplasms: a prospective randomized noninferiority trial. Endoscopy 2013;45:98-105.

8. Kudo SE, Misawa M, Wada Y, et al. Endocytoscopic microvasculature evaluation is a reliable new diagnostic method for colorectal lesions (with video). Gastrointest Endosc 2015;82:912-923.
9. Hosoe N, Kobayashi T, Kanai T, et al. In vivo visualization of trophozoites in patients with amoebic colitis by using a newly developed endocytoscope. Gastrointest Endosc 2010;72:643-646.

10. Bessho R, Kanai T, Hosoe N, et al. Correlation between endocytoscopy and conventional histopathology in microstructural features of ulcerative colitis. J Gastroenterol 2011;46:1197-1202.

11. Nishiyama S, Oka S, Tanaka S, et al. Clinical usefulness of endocytoscopy in the remission stage of ulcerative colitis: a pilot study. J Gastroenterol 2015;50:1087-1093.

12. ASGE Technology Committee. Confocal laser endomicroscopy. Gastrointest Endosc 2014;80:928-938.

13. Wallace MB, Meining A, Canto MI, et al. The safety of intravenous fluorescein for confocal laser endomicroscopy in the gastrointestinal tract. Aliment Pharmacol Ther 2010;31:548-552.

14. Becker V, von Delius S, Bajbouj M, Karagianni A, Schmid RM, Meining A. Intravenous application of fluorescein for confocal laser scanning microscopy: evaluation of contrast dynamics and image quality with increasing injection-to-imaging time. Gastrointest Endosc 2008;68:319323.

15. Kiesslich R, Burg J, Vieth M, et al. Confocal laser endoscopy for diagnosing intraepithelial neoplasias and colorectal cancer in vivo. Gastroenterology 2004;127:706-713.

16. Meining A, Saur D, Bajbouj M, et al. In vivo histopathology for detection of gastrointestinal neoplasia with a portable, confocal miniprobe: an examiner blinded analysis. Clin Gastroenterol Hepatol 2007;5:12611267.

17. Hurlstone DP, Baraza W, Brown S, Thomson M, Tiffin N, Cross SS. In vivo real-time confocal laser scanning endomicroscopic colonoscopy for the detection and characterization of colorectal neoplasia. Br J Surg 2008;95:636-645

18. Sanduleanu S, Driessen A, Gomez-Garcia E, Hameeteman W, de Bruïne A, Masclee A. In vivo diagnosis and classification of colorectal neoplasia by chromoendoscopy-guided confocal laser endomicroscopy. Clin Gastroenterol Hepatol 2010;8:371-378.

19. De Palma GD, Staibano S, Siciliano S, et al. In vivo characterisation of superficial colorectal neoplastic lesions with high-resolution probebased confocal laser endomicroscopy in combination with video-mosaicing: a feasibility study to enhance routine endoscopy. Dig Liver Dis 2010;42:791-797.

20. Buchner AM, Shahid MW, Heckman MG, et al. Comparison of probebased confocal laser endomicroscopy with virtual chromoendoscopy for classification of colon polyps. Gastroenterology 2010;138:834-842.

21. Shahid MW, Buchner AM, Heckman MG, et al. Diagnostic accuracy of probe-based confocal laser endomicroscopy and narrow band imaging for small colorectal polyps: a feasibility study. Am J Gastroenterol 2012;107:231-239

22. Shahid MW, Buchner AM, Coron E, et al. Diagnostic accuracy of probe-based confocal laser endomicroscopy in detecting residual colorectal neoplasia after EMR: a prospective study. Gastrointest Endosc 2012;75:525-533.

23. Gómez V, Shahid MW, Krishna M, Heckman MG, Crook JE, Wallace MB. Classification criteria for advanced adenomas of the colon by using probe-based confocal laser endomicroscopy: a preliminary study. Dis Colon Rectum 2013;56:967-973.

24. Wang KK, Carr-Locke DL, Singh SK, et al. Use of probe-based confocal laser endomicroscopy (pCLE) in gastrointestinal applications. A consensus report based on clinical evidence. United European Gastroenterol J 2015;3:230-254.

25. Neumann H, Vieth M, Atreya R, et al. Assessment of Crohn's disease activity by confocal laser endomicroscopy. Inflamm Bowel Dis 2012;18:2261-2269.

26. Li CQ, Xie XJ, Yu T, et al. Classification of inflammation activity in ulcerative colitis by confocal laser endomicroscopy. Am J Gastroenterol 2010;105:1391-1396. 